\title{
Medical Acute Complications of Intracerebral Hemorrhage in Young Adults
}

\author{
Riku-Jaakko Koivunen, ${ }^{1}$ Elena Haapaniemi, ${ }^{1}$ Jarno Satopää, ${ }^{2}$ Mika Niemelä, ${ }^{2}$ \\ Turgut Tatlisumak, ${ }^{1}$ and Jukka Putaala ${ }^{1}$ \\ ${ }^{1}$ Department of Neurology, Helsinki University Central Hospital, Haartmaninkatu 4, P.O. Box 340, 00290 Helsinki, Finland \\ ${ }^{2}$ Department of Neurosurgery, Helsinki University Central Hospital, 00290 Helsinki, Finland
}

Correspondence should be addressed to Riku-Jaakko Koivunen; riku.koivunen@helsinki.fi

Received 29 November 2014; Revised 19 January 2015; Accepted 20 January 2015

Academic Editor: David S. Liebeskind

Copyright (C) 2015 Riku-Jaakko Koivunen et al. This is an open access article distributed under the Creative Commons Attribution License, which permits unrestricted use, distribution, and reproduction in any medium, provided the original work is properly cited.

\begin{abstract}
Background. Frequency and impact of medical complications on short-term mortality in young patients with intracerebral hemorrhage (ICH) have gone unstudied. Methods. We reviewed data of all first-ever nontraumatic ICH patients between 16 and 49 years of age treated in our hospital between January 2000 and March 2010 to identify medical complications suffered. Logistic regression adjusted for known ICH prognosticators was used to identify medical complications associated with mortality. Results. Among the 325 eligible patients (59\% males, median age 42 [interquartile range 34-47] years), infections were discovered in $90(28 \%)$, venous thrombotic events in $13(4 \%)$, cardiac complications in 4 (1\%), renal failure in 59 (18\%), hypoglycemia in 15 (5\%), hyperglycemia in 165 (51\%), hyponatremia in $146(45 \%)$, hypernatremia in 91 (28\%), hypopotassemia in $104(32 \%)$, and hyperpotassemia in 27 (8\%). Adjusted for known ICH prognosticators and diabetes, the only independent complication associated with 3-month mortality was hyperglycemia (plasma glucose $>8.0 \mathrm{mmol} / \mathrm{L}$ ) (odds ratio: 5.90, 95\% confidence interval: $2.25-15.48, P<0.001)$. Three or more separate complications suffered also associated with increased mortality $(7.76,1.42-42.49, P=0.018)$. Conclusions. Hyperglycemia is a frequent complication of ICH in young adults and is independently associated with increased mortality. However, multiple separate complications increase mortality even further.
\end{abstract}

\section{Introduction}

Nontraumatic intracerebral hemorrhage ( $\mathrm{ICH}$ ) is a major cause of morbidity and mortality worldwide caused by a variety of different etiologies and with a case fatality at 90 days of around 35\% [1-3]. So far, treatment of ICH has been largely supportive with aims to prevent further brain injury and prevent and treat associated medical and neurological complications, such as hypertension, hyperglycemia, infections, increased intracranial pressure, seizures, electrolyte disturbances, and venous thrombotic events, which may add even further harmful effects to an already lethal disease [4]. Pulmonary complications, such as pneumonia and pulmonary embolism, and urinary tract infections have previously been reported to increase mortality, morbidity, and length-of-stay in hospital among patients with ICH [58]. Hyperglycemia has also been reported to worsen the outcome after ICH $[9,10]$.

The roles of medical complications have not yet been investigated among young adults with ICH in the few studies executed in young patients with ICH [11-18]. The young patients have different risk factors, etiology, and outcome, they have fewer coexisting chronic diseases than older ICH patients, and so their array of medical complications and their impact on outcome may differ as well [19]. We aimed to describe the in-hospital medical complications in young adult patients with first-ever ICH due to any cause and to study their impact on short-term mortality. 


\section{Methods}

This study is based on the Helsinki ICH in the Young Study, a retrospective analysis of all the consecutive patients with a nontraumatic first-ever ICH occurring at the age of 16 to 49 years treated in the Helsinki University Central Hospital (HUCH) between January 1, 2000, and March 31, 2010 [19]. This study has been approved by institutional authorities. Consent for registration was not required by the Finnish legislation as this was a registry-based study with no patient contact.

2.1. Patient Selection. Eligible patients were selected out of all the patients who at any time during their hospital stay or outpatient visit had an ICD-10 (International Classification of Diseases, 10th Revision) diagnosis code of Q28.1, Q28.3, I60.8, I61, I67.3, I67.4, I67.5, I67.6, I67.7, I67.8, I67.9, I68, or I78 through screening their brain scans. Patients with ICH due to trauma or secondary to subarachnoid hemorrhage or ischemic stroke were excluded, as were patients without available brain CT or MRI scans.

2.2. Data Retrieval. A retrospective chart review including patient medical records, laboratory database, and imaging database was executed. All patients had been initially seen by a neurologist or a neurosurgeon. NIH Stroke Scale (NIHSS) was used to assess stroke severity. Hematoma volumes were calculated with the ABC/2 method [20]. Presence of intraventricular extension of hemorrhage (IVH) was recorded but not included in volume calculations. Hematoma location was classified as lobar, deep, infratentorial, or mixed [21]. We recorded neurosurgical hematoma evacuation operations performed. Use of nasogastric tube, intubation, tracheostomy, central venous catheter, urinary tract catheter, and antibiotic treatment was recorded. Furthermore, use of bacterial culture sample from cerebrospinal fluid, urinary tract catheter, and tracheostomy or intubation tube was recorded.

2.3. Medical Complications. We recorded medical complications patients suffered any time during their acute hospital stay due to $\mathrm{ICH}$, including infections, venous thrombotic events, cardiac complications, renal failure, hypoglycemia, hyperglycemia, and sodium or potassium imbalances.

We considered as respiratory infections both pneumonias verified as infiltrate in chest radiograph together with fever, aural temperature $>38^{\circ} \mathrm{C}$, and tracheal infections verified by bacterial growth in tracheal culture in intubated or tracheostomized patients [5]. Septicemia was defined by bacterial growth in blood culture together with fever. Urinary tract infections (UTI) were registered by positive urine dipstick for nitrate or pyuria together with bacterial growth in urine sample culture. Meningitis was recorded by bacterial growth in cerebrospinal fluid sample accompanied by fever. Gastrointestinal infection was diagnosed by diarrhea or peritonitis verified in laparotomy.

Deep venous thrombosis (DVT) was recorded only when verified by ultrasound imaging of veins, and pulmonary embolism (PE) had to be verified by pulmonary CT angiography imaging. Cardiac complications included first-ever atrial fibrillation, atrial flutter, and ventricular fibrillation diagnosed by electrocardiogram (ECG) or myocardial infarction verified by ECG and elevated myocardial enzyme levels [22].

Hyperglycemia was defined as plasma glucose $>8.0 \mathrm{mmol} / \mathrm{L}(>144 \mathrm{mg} / \mathrm{dL})$ at any time during acute hospital ward. Hypoglycemia was defined as plasma glucose $<4.0 \mathrm{mmol} / \mathrm{L}(72 \mathrm{mg} / \mathrm{dL})$ [23]. Renal failure was defined as plasma creatine level $>100 \mu \mathrm{mol} / \mathrm{L}$ measured by enzymatic method [24]. Highest and lowest plasma sodium and potassium levels during hospital stay were recorded and were defined as normal by the range of values used by the Central Laboratory of Helsinki and Uusimaa Hospital District (http:// www.hus.fi/en/medical-care/laboratory): $\quad 136-145 \mathrm{mmol} / \mathrm{L}$ (313-333 mg/dL) for sodium and 3.3-5.0 mmol/L (12.9$19.6 \mathrm{mg} / \mathrm{dL}$ ) for potassium.

Our written institutional stroke treatment guidelines have recommended treatment of hyperglycemia with subcutaneous insulin every hour until level of plasma glucose $<8.0 \mathrm{mmol} / \mathrm{L}$ is reached. Intravenous insulin is used in treatment-resistant cases. Intravenous fluids with calculated percentage of sodium and potassium are administered to patients routinely to correct sodium or potassium disturbances if present.

2.4. Outcome Data. The primary outcome measure was 3month all-cause mortality. Mortality data was obtained from Statistics Finland (census date 25 June 2013). No patient was lost to follow-up. Discharge to rehabilitation center or home was recorded.

2.5. Statistical Analyses. Normality of continuous variables was tested. Results were analyzed in demographic subgroups of patients between 16 and 29, 30 and 39, and 40 and 49 years of age. Comparisons were done between patients receiving conservative or operative treatment. Categorical variables were compared with Chi square and Fisher's exact test. MannWhitney $U$-test was used to compare continuous variables with a skewed distribution between 2 and Kruskal-Wallis test between $>2$ groups. To study the impact of medical complications on mortality, we constructed a binary logistic regression model using complications associated with 3month mortality in univariate analysis with adjustment for covariates known to associate with mortality in general and young ICH patients [16, 25-27]. These covariates included age, gender, hematoma volume, infratentorial hematoma location, presence of intraventricular hemorrhage, NIHSS score, and hematoma evacuation. Urinary tract infection was excluded from this analysis due to possible observational bias. Because preexisting diabetes and hyperglycemia are intercorrelated, we forced diabetes in the model. To illustrate the additive impact of multiple different complications, we also performed a multivariable analysis with number of complications (score) as a covariate instead of individual complications. Backward likelihood-ratio method was used in all regression analyses. A two-sided $P$ value $<0.05$ was considered significant. All analyses used SPSS 21 for Windows (IBM Inc., Armonk, NY, USA). 
TABLE 1: Baseline characteristics of young ICH patients treated in Helsinki University Central Hospital between January, 2000, and March, $2010(N=325)$.

\begin{tabular}{lc}
\hline Factor & \\
\hline Males & $200(59.5)$ \\
Age & $42(34-47)$ \\
Risk factors & \\
$\quad$ Hypertension & $99(30.5)$ \\
$\quad$ Diabetes & $28(8.6)$ \\
Hematoma characteristics & \\
$\quad$ Hematoma volume $(\mathrm{mL})$ & $11(3-36)$ \\
$\quad$ Intraventricular hemorrhage & $117(36.0)$ \\
$\quad$ Infratentorial location & $52(16.0)$ \\
NIH Stroke Scale score at arrival & $8(2-19)$ \\
Etiology & \\
$\quad$ Hypertensive microangiopathy & $84(25.8)$ \\
$\quad$ Structural & $84(25.8)$ \\
$\quad$ Other & $52(16.0)$ \\
$\quad$ Unknown & $105(32.3)$ \\
Hematoma evacuation & $102(31.4)$ \\
Highest plasma glucose $(\mathrm{mmol} / \mathrm{L})$ & $8.1(6.5-10.1)$ \\
Lowest plasma glucose $(\mathrm{mmol} / \mathrm{L})$ & $5.5(4.9-6.2)$ \\
Highest plasma sodium $(\mathrm{mmol} / \mathrm{L})$ & $143(141-147)$ \\
Lowest plasma sodium $(\mathrm{mmol} / \mathrm{L})$ & $137(134-139)$ \\
Highest plasma potassium $(\mathrm{mmol} / \mathrm{L})$ & $4.3(4.0-4.6)$ \\
Lowest plasma potassium $(\mathrm{mmol} / \mathrm{L})$ & $3.5(3.2-3.7)$ \\
Highest plasma creatinine $(\mu \mathrm{mol} / \mathrm{L})$ & $75(62-92)$ \\
Lowest plasma creatinine $(\mu \mathrm{mol} / \mathrm{L})$ & $59(47-74)$ \\
\hline Data &
\end{tabular}

Data are $n$ (\%) or median (interquartile range).

\section{Results}

Out of 1325 patients screened, a total of 325 patients were eligible for the study (200 males, 59.5\%) with median age of 42 [IQR 34-47] years, 42 [34-46] for males, and 44 [3347] for females $(P=0.471)$. Reasons for exclusion included lack of ICH $(n=765)$, subarachnoid hemorrhage $(n=78)$, traumatic cause of ICH $(n=57)$ ICH being recurrent $(n=$ $38)$, patient records missing $(n=53)$, being initially treated in another hospital $(n=18)$, incorrect primary diagnosis of ICH $(n=8,0.6 \%)$, and subdural hemorrhage $(n=5)$. Median length of acute hospital stay was 13 days (IQR 5-18). Baseline data of the study population appear in Table 1.

Hyperglycemia was the most frequent complication $(50.8 \%)$, followed by hyponatremia $(44.9 \%)$, hypopotassemia (32.0\%), and hypernatremia (28.0\%). Respiratory infection was the most common of infections (Table 2). Regarding gender differences, both respiratory infections $(31.1 \%$ versus $16.7 \%, P=0.003)$ and renal failure $(24.4 \%$ versus $9.1 \%$, $P<0.001)$ were more common among males than females. Prevalence of renal failure also increased with age $(3.5 \%$, $21.5 \%$, and $21.2 \%$ in age groups of $16-29,30-39$, and $40-49$ years, resp., $P=0.007)$. No clear correlation was found with other complications and sex or age.
Operative treatment was initiated in $111(34.2 \%)$ patients, of which 102 (31.4\%) underwent hematoma evacuation, 31 (9.5\%) ventricular drainage, and 6 (1.8\%) decompressive craniectomy. Respiratory infections, meningitis, hyperglycemia, and electrolyte disturbances with the exception of hyperpotassemia were significantly more common among those who received surgical treatment (Table 2). Furthermore, use of endotracheal intubation (108 [97.3\%] versus 62 [29.0\%], $P<0.001$ ), tracheostomy (31 [27.9\%] versus 7 [3.3\%], $P<0.001)$, nasogastric tube (72 [64.9\%] versus 45 [21.0\%], $P<0.001$ ), central vein catheter (58 [52.3\%] versus 15 [7.0\%], $P<0.001)$, and administration of antibiotics (82 [73.9\%] versus 85 [39.7\%]) were more frequent in patients undergoing surgical treatment. Cerebrospinal fluid sampling (38 [34.2\%] versus 16 [7.5\%], $P<0.001$ ) and tracheal bacterial culture sampling (44 [39.6\%] versus 17 [7.9\%], $P<$ 0.001 ) were also more frequently performed on patients receiving operative treatment, while there was no statistically significant difference with urine sampling (69 [62.2\%] versus 153 [71.5\%], $P=0.086)$.

During the acute phase, none of our patients suffered from ischemic heart event. Arrhythmias recorded included one brief ventricular fibrillation in a patient who was successfully resuscitated. One flutter and two first-ever atrial fibrillation cases were also diagnosed during the hospital stay.

Arrhythmia, renal failure, hyperglycemia, and hypernatremia associated with increased 3-month mortality in univariate analysis (Table 3 ). In the logistic regression model with known prognosticators and diabetes adjusted for, the only independent complication associated with mortality at 3 months was hyperglycemia, which increased the odds of dying nearly sixfold (odds ratio 5.90, 95\% confidence interval 2.25-15.48) (Table 4). In the regression model with number of separate complications as the covariate of interest, 3 or more complications independently were associated with the risk of death by over sevenfold increase $(7.76,1.42-42.49, P=0.018)$.

\section{Discussion}

Electrolyte and glucose disturbances, in particular hyperglycemia and hyponatremia, were common in the acute phase among young patients with ICH. After adjusting for confounders, only hyperglycemia was independently associated with increased mortality. Infections were as well rather frequent, but young patients seem to survive them. Importantly, increasing number of separate complications was gradually associated with increasing odds for death at 3 months. Our observations suggest that randomized trials aiming to investigate not only treatment of hyperglycemia in $\mathrm{ICH}$ but a holistic approach to treatment of medical complications to improve patient outcomes are warranted. Further, this finding underlines one of the mechanisms by which stroke unit care reduces mortality rates as prevention and quick treatment of complications in stroke patients are a hallmark of well-organized stroke units.

Hyperglycemia has been reported to worsen the outcome after ICH, but the exact underlying mechanisms are yet to be resolved $[9,10]$. However, one study found no correlation 
TABLE 2: Overview of in-hospital medical complications and conservative or operative treatment in 325 young patients with first-ever nontraumatic intracerebral hemorrhage.

\begin{tabular}{|c|c|c|c|c|}
\hline Complication & All $(n=325)$ & Surgical treatment $(n=111)$ & Conservative treatment $(n=214)$ & $P$ value \\
\hline Any infection & $110(33.8)$ & $57(51.4)$ & $53(24.8)$ & $<0.001$ \\
\hline Sepsis & $7(2.2)$ & $3(2.7)$ & $4(1.9)$ & 0.624 \\
\hline Respiratory infection & $82(25.2)$ & $49(44.1)$ & $33(15.4)$ & $<0.001$ \\
\hline Meningitis & $2(0.6)$ & $2(1.8)$ & 0 & 0.049 \\
\hline Gastric infection & $4(1.2)$ & $1(0.9)$ & $3(1.4)$ & 0.698 \\
\hline Urinary tract infection & $40(12.3)$ & $14(12.6)$ & $26(12.1)$ & 0.904 \\
\hline Deep venous thrombosis & $11(3.4)$ & $2(1.8)$ & $9(4.2)$ & 0.256 \\
\hline Pulmonary embolism & $4(1.2)$ & 0 & $4(1.9)$ & 0.146 \\
\hline Arrhythmia & $4(1.2)$ & 0 & $4(1.9)$ & 0.146 \\
\hline Renal failure & $59(18.2)$ & $16(14.4)$ & $43(20.1)$ & 0.208 \\
\hline Hyperglycemia & $165(50.8)$ & $88(79.3)$ & $77(36.0)$ & $<0.001$ \\
\hline Hypoglycemia & $15(4.6)$ & $6(5.4)$ & $9(4.2)$ & 0.625 \\
\hline Hyponatremia & $146(44.9)$ & $66(59.5)$ & $80(37.4)$ & $<0.001$ \\
\hline Hypernatremia & $91(28.0)$ & $50(45.0)$ & $41(19.2)$ & $<0.001$ \\
\hline Hypopotassemia & $104(32.0)$ & $45(40.5)$ & $59(27.6)$ & 0.022 \\
\hline Hyperpotassemia & $27(8.3)$ & $11(9.9)$ & $16(7.5)$ & 0.482 \\
\hline
\end{tabular}

Data are $n(\%)$.

TABLE 3: Univariate analysis of in-hospital complications on 3month mortality in young patients with intracerebral hemorrhage.

\begin{tabular}{lccc}
\hline Complication & $\begin{array}{c}\text { Alive } \\
(n=270)\end{array}$ & $\begin{array}{c}\text { Dead } \\
(n=55)\end{array}$ & $P$ value \\
\hline Sepsis & $5(1.9)$ & $2(3.6)$ & 0.406 \\
Respiratory infection & $63(23.3)$ & $19(34.5)$ & 0.081 \\
Meningitis & $2(0.7)$ & 0 & 0.522 \\
Gastric infection & $4(1.5)$ & 0 & 0.364 \\
Urinary tract infection & $39(14.4)$ & $1(1.8)$ & $\mathbf{0 . 0 0 9}$ \\
Deep venous thrombosis & $10(3.7)$ & $1(1.8)$ & 0.481 \\
Pulmonary embolism & $3(1.1)$ & $1(1.8)$ & 0.665 \\
Arrhythmia & $1(0.4)$ & $3(5.5)$ & $\mathbf{0 . 0 0 2}$ \\
Renal failure & $43(15.9)$ & $16(29.1)$ & $\mathbf{0 . 0 2 1}$ \\
Hypoglycemia & $10(3.7)$ & $5(9.1)$ & 0.083 \\
Hyperglycemia & $122(45.2)$ & $43(78.2)$ & $<\mathbf{0 . 0 0 1}$ \\
Hyponatremia & $117(43.7)$ & $29(53.7)$ & 0.176 \\
Hypernatremia & $66(24.6)$ & $25(46.3)$ & $\mathbf{0 . 0 0 1}$ \\
Hypopotassemia & $81(30.2)$ & $23(42.6)$ & 0.076 \\
Hyperpotassemia & $19(7.1)$ & $8(14.8)$ & 0.063 \\
Number of complications & & & \\
0 & $64(23.7)$ & $2(3.6)$ & \\
1 & $64(23.7)$ & $1(1.8)$ & $<\mathbf{0 . 0 0 1}$ \\
2 & $50(18.5)$ & $16(29.1)$ & \\
3 or more & $92(34.1)$ & $36(65.5)$ & \\
\hline
\end{tabular}

Data are $n(\%)$.

between blood glucose and outcome after adjusting for ICH Score, and high blood glucose level was not associated with hematoma expansion or perihematomal edema [19]. Our and
TABLE 4: Logistic regression analysis of hyperglycemia and 3-month mortality in young patients with intracerebral hemorrhage.

\begin{tabular}{lcc}
\hline Factor & OR $(95 \% \mathrm{CI})$ & $P$ value \\
\hline Female & $1.78(0.71-4.49)$ & 0.220 \\
Age group & 1 & \\
$\quad 16-29$ & $0.04(0.01-0.25)$ & N.A. \\
$30-39$ & $0.27(0.09-0.81)$ & $\mathbf{0 . 0 2 0}$ \\
$\quad 40-49$ & & \\
Hematoma volume (mL) & 1 & N.A. \\
$\quad 0-30$ & $1.75(0.61-5.01)$ & 0.296 \\
$\quad 30-60$ & $2.97(0.80-10.97)$ & 0.103 \\
$\quad>60$ & $1.18(1.13-1.23)$ & $<\mathbf{0 . 0 0 1}$ \\
NIH Stroke Scale, per point & $1.44(0.57-3.60)$ & 0.441 \\
Intraventricular hemorrhage & $0.40(0.12-1.24)$ & 0.109 \\
Infratentorial location & $0.07(0.02-0.20)$ & $<\mathbf{0 . 0 0 1}$ \\
Hematoma evacuation & $1.23(0.04-37.93)$ & 0.908 \\
Arrhythmia & $1.99(0.76-5.21)$ & 0.159 \\
Renal failure & $5.90(2.25-15.48)$ & $<\mathbf{0 . 0 0 1}$ \\
Hyperglycemia & $1.33(0.50-3.54)$ & 0.563 \\
Hypernatremia & $1.54(0.40-6.01)$ & 0.533 \\
Diabetes & &
\end{tabular}

OR, odds ratio; $\mathrm{CI}$, confidence interval.

others' results indicate that better understanding of the role and mechanisms of hazard of hyperglycemia is needed. The optimal criteria and method for treatment of hyperglycemia are not solidly defined and should, therefore, be further investigated in a properly designed clinical trial $[28,29]$. Hypoglycemia in our cases likely occurred due to excessive insulin treatment. The rates of hypoglycemia among stroke patients 
have not been previously reported. In contrast to prior findings with ischemic stroke [30], no association was observed with hypoglycemia and mortality, which may result due to our relatively small patient sample. Treatment of hyperglycemia should be adjusted more precisely in order to avoid hypoglycemia.

Electrolyte disturbances may arise after brain injury due to central nervous system having a major role in controlling sodium, potassium, and water homeostasis [31-33]. Potassium disturbances and outcome after ICH have not yet been studied. Prevalence of hyponatremia among ICH patients has been reported to be $15.6 \%$, and it has been identified as a predictor of in-hospital mortality in a recent study [34]. Our findings, in contrast, suggest that hyponatremia or other individual electrolyte disturbances do not increase mortality in young $\mathrm{ICH}$ patients.

Stroke induces immunosuppression by cellular pathways, and infections are frequent after acute stroke [35]. The need for tracheostomy could potentially be predicted early in the course of ICH based on the admission GCS score, and early tracheostomy could decrease risks of prolonged endotracheal intubation [36]. In contrast to our findings with young ICH patients, respiratory infections are associated with poor outcome in general patients with ICH or ischemic stroke [5$8,37,38]$. Reason for this remains unresolved, but possible explanations include lesser risk factors and comorbidities in young patients, more aggressive or active treatment strategies for infections, and more benign course of ICH [19].

Our results on the incidence of pneumonia among patients with ICH is in accordance with previous studies $[5,8]$. Antibiotic treatment was initiated in half of our patients, which is markedly more than number of patients in which the infection site could be detected, suggesting that only a suspicion of an infection based on fever or mildly elevated inflammatory parameters frequently leads to administration of antimicrobial treatments. Alarming worldwide phenomenon of microbes becoming resistant to antibiotics raises the question whether the liberal use of antibiotics should be more restricted.

Patients with ICH are often immobilized due to impaired consciousness or motor hemiparesis increasing their risk for DVT and PE [8, 39]. Prior studies show that lowdose heparin treatment was not associated with increased hematoma growth after 48 hours of ICH [40]. However, it is yet to be decided whether more aggressive DVT surveillance is needed, since even in our series of young $\mathrm{ICH}$ patients, approximately $5 \%$ suffered from DVT or pulmonary embolism. However, neither of these showed association with higher mortality [41].

By our results, no myocardial ischemic in-hospital events were encountered and arrhythmias were considerably rare, which is in accordance with a previous report in nonselected ICH patients [22]. Although arrhythmias were more common in those dying within 3 months, no significant correlation with short-term mortality was observed after adjusting for confounders, which is probably due to the low number of patients $(n=4)$.

To our knowledge, renal failure has not previously been investigated in young patients with $\mathrm{ICH}$, but in patients with ischemic stroke both low and high glomerular filtration rates have been reported to predict long-term mortality [24]. The relatively high number of renal failure cases observed in our study reflects the criteria we used but is rarer than in general $\mathrm{ICH}$ patients. A recent study found chronic kidney failure in nearly third of general ICH patients and it was associated with higher mortality [42]. Renal failure was associated with higher risk of death in our univariate analysis but not after adjusting for other prognosticators.

Our study has limitations. It is retrospective in nature, which may cause bias in the diagnoses of infectious complications. The exact interval by days from $\mathrm{ICH}$ onset to development of complication was not recorded. It is possible that in particular more infective and thromboembolic complications may have occurred after the acute hospital period. The ascertainment of PE and DVT depended on the physician pursuing the diagnostic work-up, and clinically silent DVTs have probably also gone undetected. Our patient cohort included only young patients, which makes the setting distinct compared to general ICH cohort, for example, with respect to lower mortality. Due to low number of deaths, some of our multivariable estimates may be instable and should be interpreted with caution. Nevertheless, we believe our relatively large number of patients compensates for the disadvantages of our study design.

\section{Conflict of Interests}

The authors declare that there is no conflict of interests regarding the publication of this paper.

\section{Acknowledgments}

The authors are indebted to Marja Metso, Saija Eirola, and Jaana Koski, RNs, for their technical assistance. This work was supported by the Helsinki Biomedical Graduate School and the Helsinki University Central Hospital Research Funds.

\section{References}

[1] C.-F. Tsai, B. Thomas, and C. L. M. Sudlow, "Epidemiology of stroke and its subtypes in Chinese vs white populations," Neurology, vol. 81, no. 3, pp. 264-272, 2013.

[2] A. Meretoja, D. Strbian, J. Putaala et al., "SMASH-U: a proposal for etiologic classification of intracerebral hemorrhage," Stroke, vol. 43, no. 10, pp. 2592-2597, 2012.

[3] A. Meretoja, R. O. Roine, M. Kaste et al., "Stroke monitoring on a national level: PERFECT stroke, a comprehensive, registrylinkage stroke database in Finland," Stroke, vol. 41, no. 10, pp. 2239-2246, 2010.

[4] J. S. Balami and A. M. Buchan, "Complications of intracerebral haemorrhage," The Lancet Neurology, vol. 11, no. 1, pp. 101-118, 2012.

[5] M. Alsumrain, N. Melillo, V. A. Debari et al., "Predictors and outcomes of pneumonia in patients with spontaneous intracerebral hemorrhage," Journal of Intensive Care Medicine, vol. 28, no. 2, pp. 118-123, 2013.

[6] K. Y. A. Umarova, A. V. Chugunov, A. A. Agapov, and P. R. Kamchatnov, "Pneumonia in patients with acute brain stroke," 
Zhurnal Nevrologii i Psihiatrii imeni S.S. Korsakova, vol. 107, supplement 21, pp. 25-29, 2007.

[7] P. M. Ossou-Nguiet, B. F. Ellenga-Mbolla, A. S. W. Odzebe et al., "Impact of urinary tract and pulmonary infection on mortality after intracerebral hemorrhage in Brazzaville," World Journal of Neuroscience, vol. 3, no. 4, pp. 246-249, 2013.

[8] B. V. Maramattom, S. Weigand, M. Reinalda, E. F. M. Wijdicks, and E. M. Manno, "Pulmonary complications after intracerebral hemorrhage," Neurocritical Care, vol. 5, no. 2, pp. 115-119, 2006.

[9] S. Passero, G. Ciacci, and M. Ulivelli, "The influence of diabetes and hyperglycemia on clinical course after intracerebral hemorrhage," Neurology, vol. 61, no. 10, pp. 1351-1356, 2003.

[10] W. Feng, S. Tauhid, S. Goel, E. V. Sidorov, and M. Selim, "Hyperglycemia and outcome in intracerebral hemorrhage: from bedside to bench-more study is needed," Translational Stroke Research, vol. 3, no. 1, pp. 113-118, 2012.

[11] G. J. Toffol, J. Biller, and H. P. Adams Jr., "Nontraumatic intracerebral hemorrhage in young adults," Archives of Neurology, vol. 44, no. 5, pp. 483-485, 1987.

[12] H. Bevan, K. Sharma, and W. Bradley, "Stroke in young adults," Stroke, vol. 21, no. 3, pp. 382-386, 1990.

[13] J. L. Fuh, H. C. Liu, S. J. Wang, Y. K. Lo, and L. S. Lee, "Nontraumatic hemorrhagic stroke in young adults in Taiwan," Journal of Stroke and Cerebrovascular Diseases, vol. 4, pp. 101105, 1994.

[14] C. L. Lin and S. L. Howng, "Nontraumatic intracerebral hemorrhage in young adult," The Kaohsiung Journal of Medical Sciences, vol. 13, no. 4, pp. 237-242, 1997.

[15] A. Awada, A. Daif, T. Obeid, and S. Al Rajeh, "Nontraumatic cerebral hemorrhage in the young: a study of 107 cases," Journal of Stroke and Cerebrovascular Diseases, vol. 7, no. 3, pp. 200-204, 1998.

[16] J. L. Ruíz-Sandoval, C. Cantú, and F. Barinagarrementeria, "Intracerebral hemorrhage in young people: analysis of risk factors, location, causes, and prognosis," Stroke, vol. 30, no. 3, pp. 537-541, 1999.

[17] O. H. del Brutto, J. Sánchez, X. Campos, J. Santos, and A. Mosquera, "Non-traumatic intracerebral hemorrhage in young adults living in Guayaquil, Ecuador (South America): analysis of 151 patients," Functional Neurology, vol. 14, no. 1, pp. 21-28, 1999.

[18] S. Roditis and N. Ianovici, "Haemorrhagic stroke in young people," Romanian Neurosurgery, vol. 18, pp. 294-294, 1999.

[19] R.-J. Koivunen, J. Satopää, A. Meretoja et al., "Incidence, risk factors, etiology, severity, and short-term outcome of nontraumatic intracerebral hemorrhage in young adults," European Journal of Neurology, vol. 22, no. 1, pp. 123-132, 2015.

[20] R. U. Kothari, T. Brott, J. P. Broderick et al., "The ABCs of measuring intracerebral hemorrhage volumes," Stroke, vol. 27, no. 8, pp. 1304-1305, 1997.

[21] G. J. Falcone, A. Biffi, H. B. Brouwers et al., "Predictors of hematoma volume in deep and lobar supratentorial intracerebral hemorrhage," JAMA Neurology, vol. 70, no. 8, pp. 988-994, 2013.

[22] J. Putaala, M. Lehto, A. Meretoja et al., "In-hospital cardiac complications after intracerebral hemorrhage," International Journal of Stroke, vol. 9, no. 6, pp. 741-746, 2014.

[23] G. Ntaios, M. Egli, A. Arsovska et al., "An intravenous insulin protocol for strict glycemic control in acute ischaemic stroke," European Journal of Neurology, vol. 19, no. 3, pp. 443-451, 2012.
[24] J. Putaala, E. Haapaniemi, D. Gordin et al., "Factors associated with impaired kidney function and its impact on long-term outcome in young ischemic stroke," Stroke, vol. 42, no. 9, pp. 2459-2464, 2011.

[25] J. C. Hemphill III, D. C. Bonovich, L. Besmertis, G. T. Manley, and S. C. Johnston, "The ICH score: a simple, reliable grading scale for intracerebral hemorrhage," Stroke, vol. 32, no. 4, pp. 891-897, 2001.

[26] S.-L. Lai, S.-T. Chen, T.-H. Lee, L.-S. Ro, and S.-P. Hsu, "Spontaneous intracerebral hemorrhage in young adults," European Journal of Neurology, vol. 12, no. 4, pp. 310-316, 2005.

[27] R.-J. Koivunen, J. Satopää, E. Haapaniemi et al., "Predictors of early mortality in young adults after intracerebral hemorrhage," Stroke, vol. 45, no. 8, pp. 2454-2456, 2014.

[28] C. S. Gray, A. J. Hildreth, P. A. Sandercock et al., "Glucosepotassium-insulin infusions in the management of post-stroke hyperglycaemia: the UK Glucose Insulin in Stroke Trial (GISTUK)," The Lancet Neurology, vol. 6, no. 5, pp. 397-406, 2007.

[29] C. S. Gray, A. J. Hildreth, G. K. M. M. Alberti, and J. E. O'Connell, "Poststroke hyperglycemia: natural history and immediate management," Stroke, vol. 35, no. 1, pp. 122-126, 2004.

[30] G. Ntaios, M. Egli, M. Faouzi, and P. Michel, "J-Shaped association between serum glucose and functional outcome in acute ischemic stroke," Stroke, vol. 41, no. 10, pp. 2366-2370, 2010.

[31] K. Bradshaw and M. Smith, "Disorders of sodium balance after brain injury," Continuing Education in Anaesthesia, Critical Care and Pain, vol. 8, no. 4, pp. 129-133, 2008.

[32] K. Kusuda, Y. Saku, S. Sadoshima, I. Kozo, and M. Fujishima, "Disturbances of fluid and electrolyte balance in patients with acute stroke," Japanese Journal of Geriatrics, vol. 26, no. 3, pp. 223-227, 1989.

[33] M. N. Alam, M. J. Uddin, K. M. Rahman et al., "Electrolyte changes in stroke," Mymensingh Medical Journal, vol. 21, no. 4, pp. 594-599, 2012.

[34] J. B. Kuramatsu, T. Bobinger, B. Volbers et al., "Hyponatremia is an independent predictor of in-hospital mortality in spontaneous intracerebral hemorrhage," Stroke, vol. 45, no. 5, pp. 12851291, 2014.

[35] Á. Chamorro, A. Meisel, A. M. Planas, X. Urra, D. Van De Beek, and R. Veltkamp, "The immunology of acute stroke," Nature Reviews Neurology, vol. 8, no. 7, pp. 401-410, 2012.

[36] S. Yaghi, P. Moore, B. Ray, and S. G. Keyrouz, "Predictors of tracheostomy in patients with spontaneous intracerebral hemorrhage," Clinical Neurology and Neurosurgery, vol. 115, no. 6, pp. 695-698, 2013.

[37] X. Fu, K. S. Wong, J. W. Wei et al., "Factors associated with severity on admission and in-hospital mortality after primary intracerebral hemorrhage in China," International Journal of Stroke, vol. 8, no. 2, pp. 73-79, 2013.

[38] M. Al-Khaled and J. Eggers, "QugSS2 Study. Prognosis of intracerebral hemorrhage after conservative treatment," Journal of Stroke and Cerebrovascular Diseases, vol. 23, no. 2, pp. 230234, 2014.

[39] U. Dickmann, E. Voth, H. Schicha, T. Henze, H. Prange, and D. Emrich, "Heparin therapy, deep-vein thrombosis and pulmonary embolism after intracerebral hemorrhage," Klinische Wochenschrift, vol. 66, no. 23, pp. 1182-1183, 1988.

[40] D. N. Orken, G. Kenangil, H. Ozkurt et al., "Prevention of deep venous thrombosis and pulmonary embolism in patients with acute intracerebral hemorrhage," Neurologist, vol. 15, no. 6, pp. 329-331, 2009. 
[41] A. Y. Zubkov and E. F. M. Wijdicks, "Deep venous thrombosis prophylaxis in cerebral hemorrhage," Reviews in Neurological Diseases, vol. 6, no. 1, pp. 21-25, 2009.

[42] B. Ovbiagele, L. H. Schwamm, E. E. Smith et al., "Hospitalized hemorrhagic stroke patients with renal insufficiency: clinical characteristics, care patterns, and outcomes," Journal of Stroke and Cerebrovascular Diseases, vol. 23, pp. 2265-2273, 2014. 


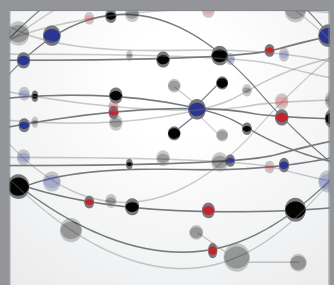

The Scientific World Journal
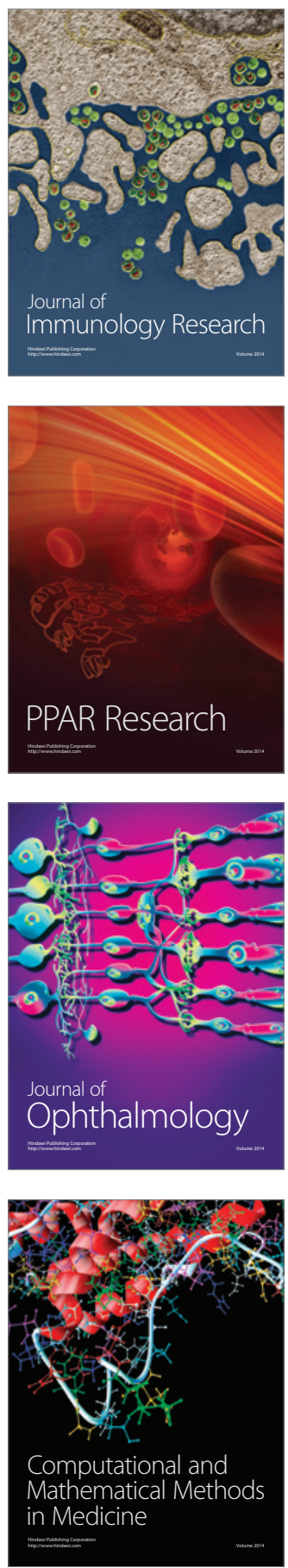

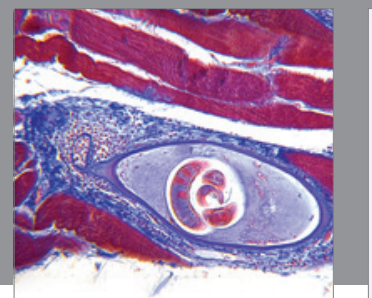

Gastroenterology

Research and Practice
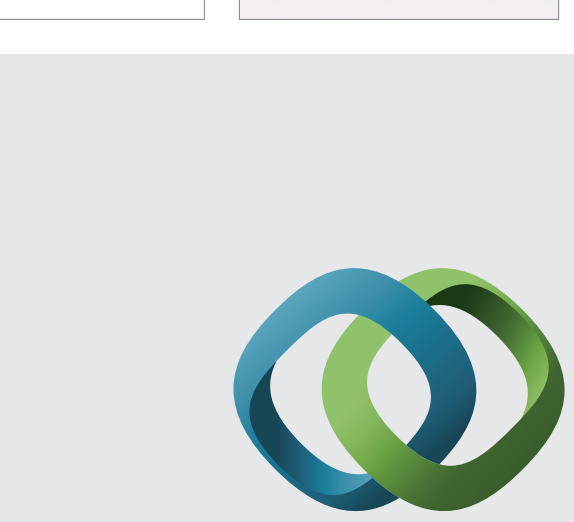

\section{Hindawi}

Submit your manuscripts at

http://www.hindawi.com
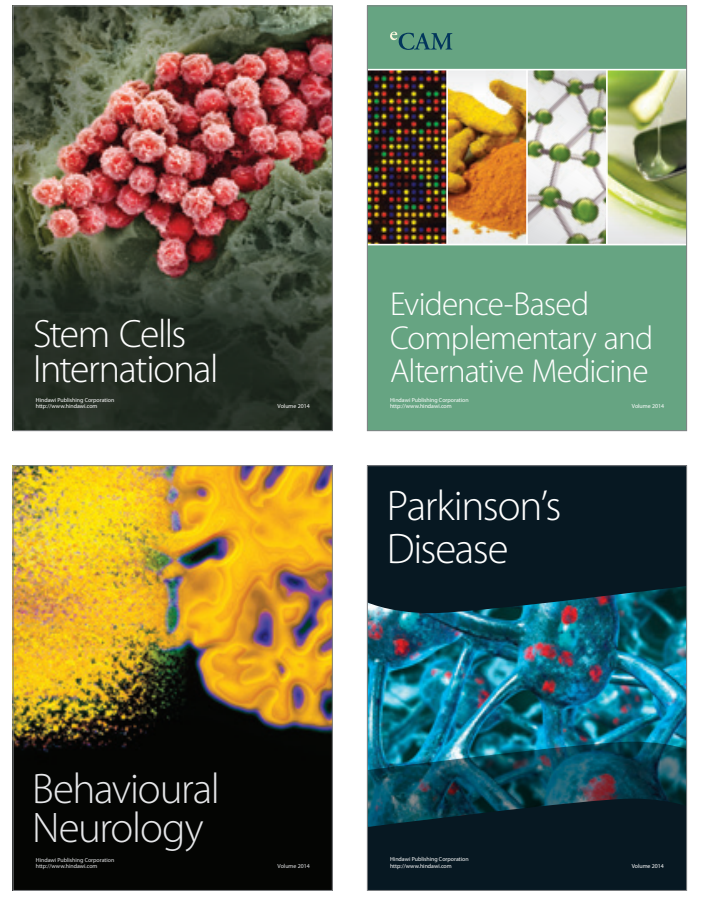
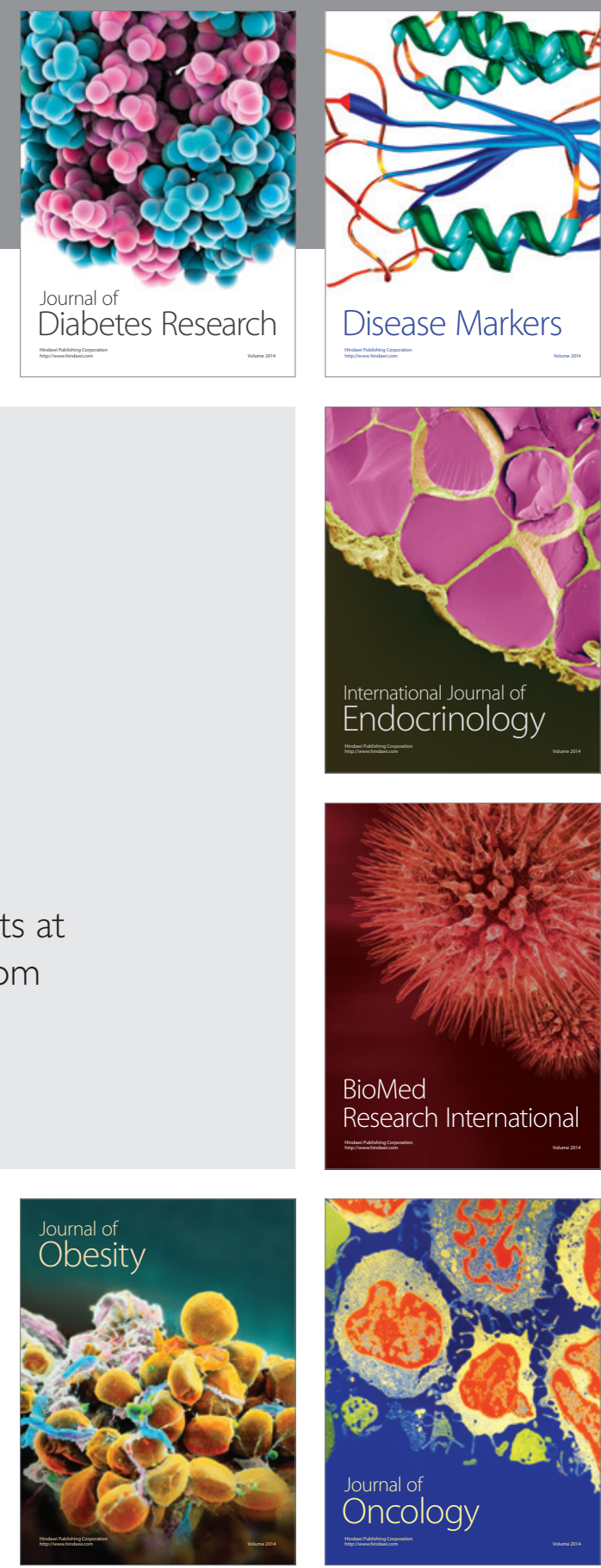

Disease Markers
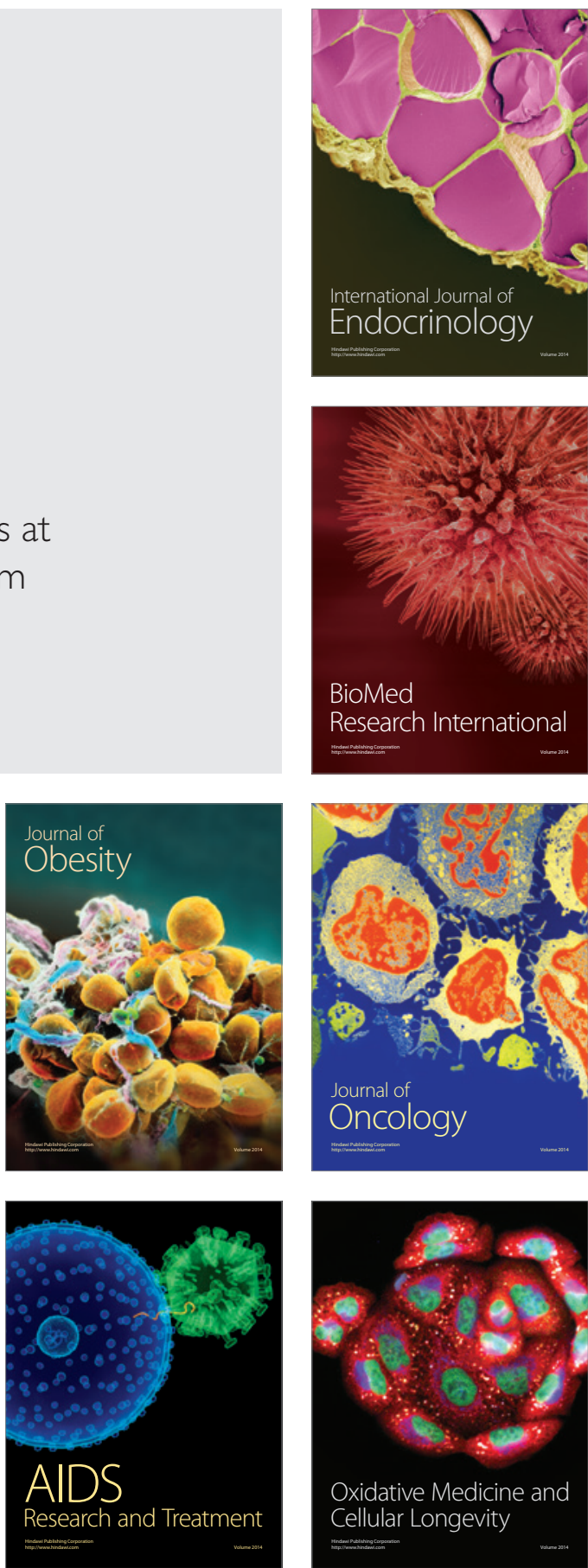